\title{
Beamforming for Direction-of-Arrival (DOA) Estimation-A Survey
}

\author{
V Krishnaveni, PhD. \\ Associate Professor \\ ECE Department \\ PSG College of Technology
}

\author{
T Kesavamurthy, PhD. \\ Assistant Professor (Sr.) \\ ECE Department \\ PSG College of Technology
}

\author{
Aparna.B \\ PG Student \\ ECE Department \\ PSG College of Technology
}

\begin{abstract}
Direction-of-Arrival (DOA) estimation plays a vital role in many applications. Beamforming is the most prominent technique to estimate DOA. In this survey, a study of various beamforming techniques and algorithms to estimate the direction of arrival of a signal is made. An assessment on the background robust algorithms using Nyquist sampling rate and its Compressive sensing alternative is done. It is known that Bearing estimation algorithms obtain only a small number of direction of arrivals (DOAs) within the entire angle domain, when the sources are spatially sparse. Hence, it may be concluded that, the methods those specifically exploits this spatial sparsity property is advantageous. These methods use a very small number of measurements in the form of random projections of the sensor data along with one full waveform recording at one of the sensors.
\end{abstract}

\section{Keywords}

Beamforming, Direction-of-Arrival, Array processing, MVDR, MUSIC, Root-MUSIC, ESPRIT, Spatial sparsity, Compressive Sensing.

\section{INTRODUCTION}

The problem of estimating the wave number or angle of arrival of a plane wave is referred to as direction finding or DOA estimation problem. It has a large application in radar, sonar, seismic systems, electronic surveillance, medical diagnosis and treatment, radio astrology and other areas. Because of its widespread applications and difficulty of obtaining an optimum estimator, the topic has a received a significant amount of attention over the last several decades. Several methods exist to address the problem of estimating the direction-of-arrivals (DOAs) of multiple sources using the signals received at the sensors The application of the array processing requires either the knowledge of a reference signal or the direction of the desired signal source to achieve its desired objectives. Antenna arrays are widely used to solve direction finding.

Beamforming is used along with an array of antennas/sensors to transmit/receive signals to/from a specified spatial direction in the presence of interference and noise. Hence it acts as a spatial filter [1]. It is a classic yet continuously developing field that has enormous practical applications. In the last decade, there has been renewed interest in beamforming driven by applications in wireless communications, where multiantenna techniques have emerged as one of the key technologies to accommodate the explosive growth of the number of users and rapidly increasing demands for new high data-rate services.

The techniques for estimating the directions of arrival of signals using an antenna array have been booming in recent years. Many methods exist and are classified according to the technique used, the information they require (external or not) and finally the criterion used (conventional methods, projection on the noise or source subspace, maximum likelihood method, etc. A receive beamformer is commonly used to estimate the signal arriving from a spe $\tau$ cific direction in the presence of noise and interfering signals. In a receive beamformer, the output of the array of sensors are linearly combined using spatial filter coefficients(weight vector) so that the signals coming from a desired direction are passed to the beamformer output undistorted, while signals from other directions are attenuated.

With a central focus on bearing estimation, the prime objective here is to locate the source of transmitted communication/ radar signal. Through this paper, a detailed literature survey is made on the various bearing estimation techniques and algorithms till date. These many estimation algorithms which are available in the literature have different capabilities and limitations [2]-[3]. The DOA estimation problem in some cases, is first solved by estimation methods of the number of sources [4]-[5]-[6] and then applying a high-resolution method to estimate the angular position of these sources. These high-resolution methods are known to be more robust than conventional techniques.

The most general beamforming techniques include conventional as well as adaptive beamformers. For the conventional non-adaptive beamformers, the weight vector for a specific direction of arrival (DOA) depends on the array response alone and can be pre-calculated, independent of the received data. Hence they are data independent beamformers and they present a constant response for all signal/interference scenarios. The adaptive beamformers are data-dependent since the weight vectors are calculated as a function of the incoming data to optimize the performance subject to various constraints [2]. They have better resolution and much better interference rejection capability than the data-independent beamformers.

However, in practical array systems, traditional adaptive beamforming algorithms are known to degrade, if some of exploited assumptions on the environment, sources, or sensor array become wrong or imprecise. Similar types of degradation can occur when the signal array response is known exactly, but the training sample size is small. Therefore, the robustness of adaptive beamforming techniques against environmental and array imperfections and uncertainties remains one of the key issues.

The commonly used algorithms, acquires the source signals at the Nyquist rate and transmits all measurements to a central processor in order to estimate just a small number of source bearings. The communication load between sensors can be drastically reduced, however, by exploiting spatial sparsity, i.e., the fact that the number of sources we are trying to find is much less than the total number of possible 
source bearings. Compressive sensing (CS) is a recent technique which accomplishes with the concept of sparsity proving to be advantageous over the popular Nyquist Sampling rate.

In this paper, a detailed literature survey on the commonly used DOA estimation techniques, is made first, and later moving on to the Compressive Sensing (CS) technique which may be more advantageous for DOA estimation.

\section{BEAMFORMING}

A conventional beamformer has a structure shown in Figure 1 , where the weights are pre-calculated and are independent of the incoming data. The weights are basically the delay encountered in each sensor due to path difference, so that the outputs of spatially distributed sensors are coherently summed to improve signal reception in the presence of noise.

Consider a linear array of $\mathrm{M}$ sensors, with a uniform inter sensor spacing (d). The sensors spatially sample the signal field at the sensor locations. We assume that a narrow-band source $\mathrm{x}(\mathrm{t})$ is impinging upon the array from angle $\theta$, with respect to the array axis and that the source is in the far-field of the array. If $x(t)$ is the signal that would be received at the origin of the coordinate system, then the signal received by the $\mathrm{M}$ sensors of the array at time instant $\mathrm{t}, \mathrm{x}_{1}(\mathrm{t}) \ldots . . \mathrm{x}_{\mathrm{M}}(\mathrm{t})$ can be written as the vector $\mathrm{x}(\mathrm{t})$,

$$
x(t)=\left[x\left(t-\tau_{1}\right), x\left(t-\tau_{2}\right), \ldots . . x\left(t-\tau_{M}\right)\right]^{T}
$$

where $\tau_{1, \ldots .} \tau_{\mathrm{M}}$ is the time delay occurred for the signal received at each of the sensors due to the path difference.

For a linear array with uniform sensor spacing d, the time delay between the adjacent sensors is given by

$$
\tau=(d \cos \theta) / c
$$

where $c$ is the velocity of medium.

The time delay between 1 st and $\mathrm{M}^{\text {th }}$ sensor will be

$$
\tau_{M}=((M-1) d \cos \theta) / c
$$

If $\mathrm{w}_{1} \ldots \mathrm{w}_{\mathrm{M}}$ are the weights to be multiplied to each of the sensor outputs which is collectively represented by the vector $\mathrm{w}$, then the scalar beamformer output $y(\mathrm{t})$ generated by the linear combination of $\mathrm{x}(\mathrm{t})$ and $\mathrm{w}$ can be represented as

$$
y(t)=w^{H} x(t)
$$

Different beamforming approaches correspond to different choices of the weighting vector $\mathrm{w}$. The conventional nonadaptive beamformers are data-independent in the sense that the weight vectors can be pre-calculated independent of the incoming data. These beamformers include the delay-andsum approach as well as methods based on various weight vectors for sidelobe control.

\subsection{Conventional Time domain \\ Beamformer}

The time delay between the sensor data due to the sensor array geometry is compensated in time domain by giving appropriate time delays to the sensor data and then summing it together. In a broadband time domain beamformer, interpolation filters are used at each sensor to provide the true time delay for the broadband of frequencies. The filtered outputs of all sensors are summed to form the beamformer output.

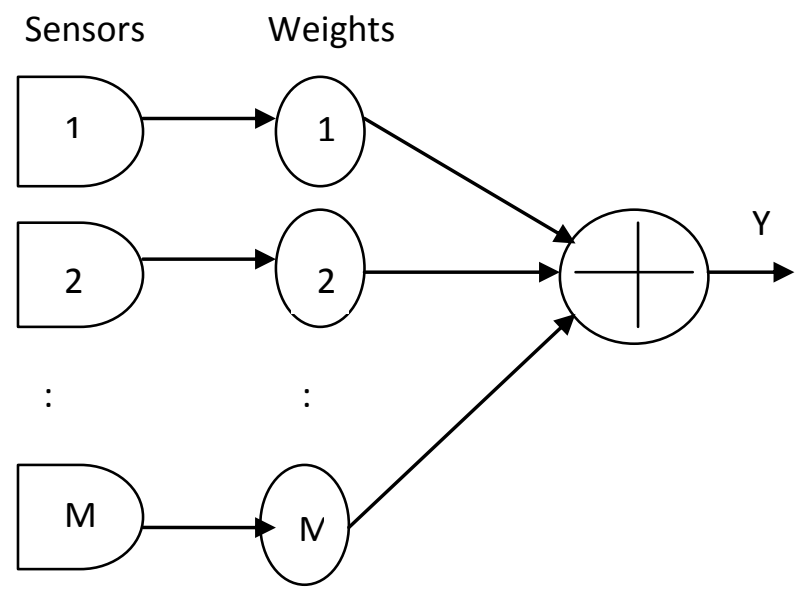

Figure 1. Conventional Beamformer

\subsection{Conventional Frequency domain Beamformer}

For a narrowband signal, the delay $\tau_{\mathrm{n}}$ is approximated by a phase shift. Hence the received sensor array data vector in frequency domain can be written as

$$
X(\omega)=X(\omega) s(\omega, \theta)
$$

where $X(\omega)$ is the Fourier transform of $x(t) . X(\omega)$ is the vector formed by the Fourier transform of sensor array data $\mathrm{x}(\mathrm{t})$ and $\mathrm{s}(\omega, \theta)$ which is given by

$$
s(\omega, \theta)=\exp \left(-j \omega\left[\tau_{1}, \tau_{2}, \ldots . . \tau_{M}\right]\right)
$$

is called the array manifold vector or the steering vector which incorporates all the spatial characteristics of the array. In a narrow band frequency domain beamformer, a beam is formed in a required direction by giving appropriate phase shifts to the sensor data to compensate for the phase shift occurred due to the path difference to different sensors in the wave front. These phase shifted channel outputs are summed together [2]. In vector form, the output of the narrowband frequency domain beamformer can be represented as

$$
Y(\omega)=s^{H}(\omega) X(\omega) \quad(7)
$$

In a broadband frequency domain beamformer, the signal is broken up into different frequency components using FFT. The phase shift for each frequency bin is computed and provided as the steering vector. The data vector for each bin is multiplied by the corresponding steering vector and summed across bins to form the beamformer output.

\subsection{Adaptive Beamformer}

Adaptive beamforming is used for enhancing a desired signal while suppressing noise and interference at the output of an array of sensors. The aim of the adaptive beamforming is to optimize a collection of weight vectors to locate a directional source. There are different methods in arriving at this optimization problem. Figure 2 shows the structure of an adaptive beamformer. In applications where signal strength is unknown and is always present, application of linear constraints to the weight vector permits extensive control of the adaptive behavior of the beamformer. 


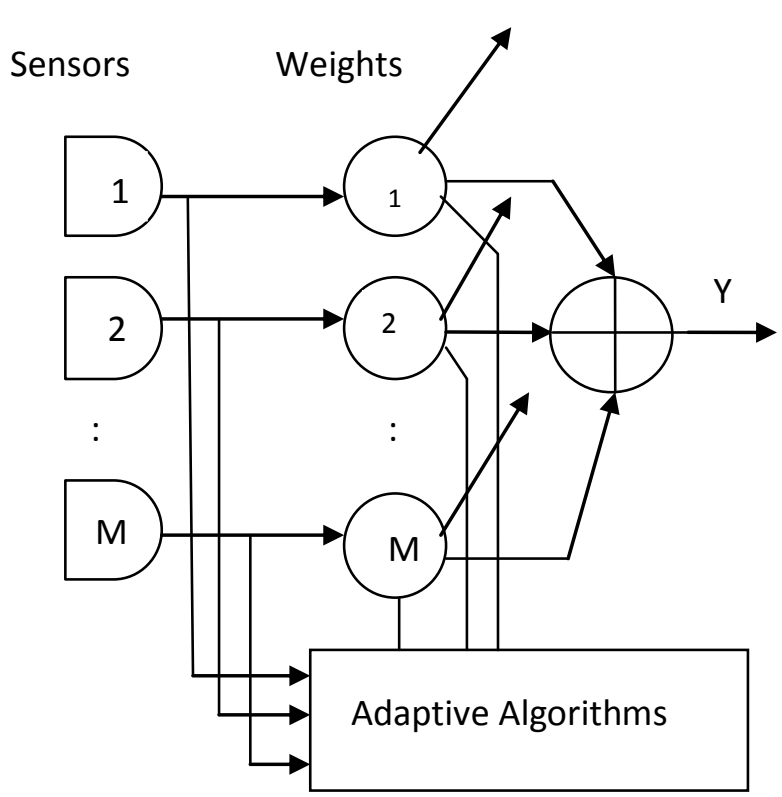

Figure 2. Adaptive Beamformer

\section{LITERATURE SURVEY}

A survey of beamformers including adaptive beamformers is given in [1]. The conditions under which the adaptive beamformer performance degrades are also seen in many papers. There exist a number of techniques to estimate the DOA of signals of interest. Here, a survey on commonly used techniques as well as algorithms is made. In general, the DOA estimation adaptive beamforming algorithms may be classified into Beamscan Algorithms, and Beamspace algorithms [2].

\subsection{Diagonal Loading}

Among the many robust adaptive beamformers proposed in the literature, diagonal loading emerges as the most widely used method due to its simplicity and its effectiveness in handling a wide variety of errors, including steering vector and finite-sample errors [4]. It is robust against finite sample errors [5]. However, a serious drawback of the diagonal loading technique is that there is no reliable way to choose the diagonal loading factor, which directly affects its performance.

\subsection{Eigenspace Based Technique}

Another popular robust adaptive beamforming technique is the eigenspace-based beamformer [6]. The key idea of this technique is to project the signal steering vector onto the estimated signal-plus-interference subspace obtained via the eigen decomposition of the sample covariance matrix. If the rank of signal-plus-interference subspace is low and if the number of interference directions, $L$ are exactly known, the eigenspace-based beamformer is known to provide excellent robustness against arbitrary steering vector errors. Unfortunately, this approach may degrade severely if the low-rank interference-plus-signal assumption is violated or if the subspace dimension $L$ is uncertain or known imprecisely. For example, in the presence of incoherently scattered (spatially dispersed) interfering sources, interferers with randomly fluctuating wave fronts, and moving interferers, the low rank interference assumption may become violated and $L$ can be uncertain. Therefore, the eigenspace-based beamformer may be not a proper method of choice in such cases. Moreover, even if the low-rank model assumption remains relevant, the eigenspace-based beamformer can be only used in scenarios where the signal-to noise ratio (SNR) is sufficiently high.

\subsection{LCMV Beamformer}

To improve the robustness of the beamformer against the DOA angle mismatch error, additional derivative constraints [7] can be imposed to the LCMV beamformer [8] so that a wider main beam can be obtained to cover all the possible directions of the signal of interest. Derivative constraints can be used at the interference directions also, under conditions when the interfering sources are rapidly moving (nonstationary). The data nonstationarity can cause these sources to may move away from the sharp notches of the adapted pattern, and this may lead to a strong degradation of the output Signal-to-Interference plus Noise ratio(SINR) . An efficient remedy for adaptive array performance in such situations is based upon artificial broadening of the null width toward the directions of interfering sources using derivative constraints [9], [10].

A robust beamformer for the most general case of an arbitrary dimension of the desired signal subspace is developed in [11], and is applicable to both the rank-one (point source) and higher rank (scattered source/fluctuating wavefront) desired signal models. The proposed robust adaptive beamformers are based on explicit modeling of uncertainties in the desired signal array response and data covariance matrix as well as worst-case performance optimization. Closed form solutions and computationally efficient online implementations of the robust algorithm are also developed in [11].

\subsection{Capon Beamforming}

In [12], the Robust Capon beamformer is proposed, where the covariance fitting formulation of the standard capon beamformer, is coupled with the constraint that the beamformer response be above some level for all the steering vectors that lie in an ellipsoid (sphere) centred on the nominal or presumed steering vector of interest. In [13], an additional norm constraint is also used to get the doubly constrained Robust Capon Beamformer.

A computationally efficient robust adaptive beamforming scheme is developed in [14], to account for the signal array response mismatch and small training sample size. It includes a quadratic inequality constraint and is implemented with gradient descent method. All the robust adaptive algorithms, surveyed till now, was for narrowband signals. But in many applications, the signals are wideband and hence robust wideband adaptive algorithms are essential. The most popular approach in the design of wideband adaptive beamformers is to decompose the received broadband signals into narrowband components (subbands) and then to apply separate narrowband beamformers to each subband [15].

\subsection{Tapped Delay Line Beamformer}

An alternate approach in the design of wide band beamformers is to use tapped delay-lines (TDLs) [16], which can form a frequency dependent response for each of the received broadband sensor signals to compensate the phase difference for different frequency components. A robust algorithm for broadband arrays was proposed in [17] using worst case optimization, where a group of constraints are imposed on sampled frequency points over the frequency range of interest to prevent the mismatched desired signal 
from being filtered out by the beamformer. High computational complexity $\&$ inability to control the response consistency to the mismatched desired signal are the demerits of [17] which are being solved in the recent method proposed in [18-19]. Here, the robustness of the wideband beamforming structure is improved using a combination of frequency invariance constraint and worst case performance optimization. It is formulated as convex optimization problem and solved using existing convex optimisation techniques.

\subsection{Algorithms}

Algorithms for estimating DOA can be classified into beamscan algorithms and subspace algorithms [2]. The beamscan algorithms form a conventional beam, scans it over the appropriate region and plots the magnitude squared of output. This estimator is referred to as the Bartlett beamformer [20-21]. MVDR, Root MVDR are the examples of this.

Subspace algorithms, are a set of algorithms, wherein the orthogonality between the signal and noise subspaces is exploited[2]. These are also referred to as high resolution subspace based algorithms. The modern high-resolution methods based on the concept of subspace, like MUSIC, Root-MUSIC and ESPRIT are among the most efficient for estimating the directions of arrival of signals using array antennas. These methods are based on the specific properties of the covariance matrix of the signal. The principle is based on the decomposition of the observation space into two subspaces: signal subspace and noise subspace. They have a high resolving power when sources are uncorrelated or weakly correlated. Their advantage is that subspaces only depend on the geometry of the network and the position of sources.

\subsubsection{MVDR Algorithms}

In Minimum Variance Distortionless Response beamformer the linear filter weights used in the beamformer are adaptively calculated depending on the environment so as to suppress the interferences to the maximum, leaving the signal of interest undistorted [22]. Here the computation of the inverse correlation matrix and its multiplication with steering vector are the most important parts in the process of optimal weight computation. The array correlation matrix (R) is a measure of the spatial correlation of the signal and noise arriving at the array. Adaptive beam forming techniques measures the array correlation matrix instead of assuming that the noise is white and Gaussian. This array correlation matrix measurement is then used to determine the spatial filter coefficients (weights). MVDR shows degraded performance compared to conventional beamformers, when there is position errors in sensors.

Root MVDR performs reasonably well above threshold, but threshold is higher than Maximum Likelihood algorithms closely spaced signals. However, the threshold of MVDR algorithm is higher than root MVDR[2]. It is used as a preliminary processor to indicate the number of plane waves impinging on the array, their approximate location, and approximate signal power. However it suffers a demerit that, in case of 2 closely spaced plane waves, algorithms will think they are single plane waves and underestimate the number of signals.

The performance of the MVDR beamformer is severely affected by the correlation between the look-direction signal and the interferences. Spatial smoothing is a technique used to alleviate the problems due to correlation where the array is divided into smaller sub arrays, and the average of the all the sub array covariance matrices are used to form a smoothed $\mathrm{R}$ matrix. In [23], it is shown that spatial smoothing progressively decorrelates the sources by diagonalizing the source covariance matrix. This decorrelation results in reduced signal cancellation and increased rejection of the coherent interference. In [24], the finite-data performance of the MVDR Beamformer with and without spatial smoothing is analyzed. It is shown that the smoothing, in addition to decorrelating the sources, can also alleviate the effects of finite-data perturbations (the covariance matrix errors due to the finite number of snapshots used for its estimation).

In the recent past, some robust algorithms with clear theoretical background have been proposed which make explicit use of an uncertainty set of the array steering vector. In [25], spherical uncertainty sets are used and in [26] ellipsoidal (including flat ellipsoidal) uncertainty sets are used. Here, the beamformer is designed to minimize the output power subject to the constraint that the beamformer response is above some level for all the steering vectors that lie in an ellipsoid (sphere) centred on the nominal or presumed steering vector of interest. This guarantees that the signal of interest, whose steering vector is expected to lie in the ellipsoid (sphere), will not be eliminated, and hence, robustness is improved. When the ellipsoid is a sphere, then the solution to the above-mentioned problem is of the diagonal loading type, where the loading level is obtained using the covariance matrix and the radius of the sphere. In the case where the ellipsoid is not a sphere or is flat, the robust beamformer takes the form of a general (i.e., not necessarily diagonal) loading of the covariance matrix. In either case, the solution is given by $(\mathrm{R}+\mathrm{Q})^{-1} \mathrm{~s}$ where $s$ denotes the nominal steering vector (in the absence of any uncertainty), and Q stands for the loading matrix.

Classes of robust MV beamforming algorithms based on optimization of worst-case performance are proposed in [2526]. The robustness of the MVDR beamformer is improved in [25] where it explicitly models an arbitrary (but bounded in norm) mismatch in the desired signal array response for point source signal models and uses worst-case performance optimization . This method is based on a convex optimization using second-order cone programming (SOCP). Although several efficient convex optimization software tools are currently available, the SOCP-based method does not provide any closed-form solution and does not have simple online implementations. In [26], the approach of [25] is extended to a more general case where, apart from the steering vector mismatch, there is a nonstationarity of the training data also. The norms of both the steering vector mismatch and the data matrix mismatch are bounded by some known constants, and the weights are calculated by optimising the worst case performance

\subsubsection{MUSIC Algorithms}

Multiple Signal Classification algorithm[27-28] uses the eigenvectors decomposition and eigenvalues of the covariance matrix of the antenna array for estimating directions-of-arrival of sources based on the properties of the signal and noise subspaces. Several variants of MUSIC like Spectral, Unitary, Root MUSIC methods have been proposed to reduce complexity, increase performance and resolution power. The advantage of Root Music is the direct calculation of the DOA by the search for zeros of a polynomial, which replaces the search for maxima[29], necessary in the case of MUSIC. This method is limited to linear antennas uniformly spaced out. But it allows a reduction in computing time and so an increase in the angular resolution by exploiting certain properties of the received signals. The principle of the Root- 
MUSIC algorithm is to form a polynomial of degree 2(M-1) and extract the roots [29]. Spectral MUSIC has less resolution capability than ROOT MUSIC[2,29]. UnitaryMUSIC [30] gives the same performance of ROOT MUSIC with an advantage of less computational complexity.

\subsubsection{ESPIRIT Algorithms}

Estimation of Signal parameters via rotational invariance technique, is based on the rotational invariance property of the signal space [31-32] to make a direct estimation of the DOA and obtain the angles of arrival without the calculation of a pseudo-spectrum on the extent of space, nor even the search for roots of a polynomial. ESPIRIT is similar to MUSIC algorithm with slight modifications. The main advantage of this method is that it avoids the heavy research of maxima of a pseudo-spectrum or a cost function (therefore a gain calculation) and the simplicity of its implementation. In addition, this technique is less sensitive to noise than MUSIC and Root-MUSIC [33]. It has been shown in [34][35] that MUSIC and ESPRIT algorithms achieve almost identical performance in the case of unmodulated sinusoids, but that ESPRIT is slightly better than MUSIC. Ultimately, ESPRIT appears less sensitive to noise than MUSIC[36].

These prevalent background algorithms acquire the source signals at the Nyquist rate and take a higher number of samples to estimate a parameter which is sparse in some other domain. Hence it is advisable to exploit this sparsity property to estimate the DOA of the desired signal. The sparsity property of signals has been utilized in a variety of applications including image reconstruction, medical imaging radar imaging, blind source separation and shape detection. In the literature, sparsity information has also been used previously for beamforming and source localization.

The concept of the spatial sparsity of sources can be exploited to accomplish source localization in arbitrary shaped sensor arrays for both narrowband and wideband signals by using a very small number of measurements, thereby improving the communication efficiency of sensor networks [37-38]. Although prior research has validated the benefits of exploiting spatial sparsity in source localization, such as improved resolution, the methods also require a high sampling rate of source signals, which increases the communication load between sensors. This is an important consideration for energy efficient wireless sensor networks. Furthermore, in some applications, data acquisition might be very expensive. A comparison of the different beamforming techniques and algorithms is given in Table 1.

Table 1. Comparison of different beamforming techniques for DOA estimation

\begin{tabular}{|l|l|l|}
\hline Technique & Merits & Demerits \\
\hline $\begin{array}{l}\text { Diagonal } \\
\text { Loading } \\
\text { Beamformer }\end{array}$ & $\begin{array}{l}\text { Robust against } \\
\text { finite sample } \\
\text { errors }\end{array}$ & $\begin{array}{l}\text { No reliable way to } \\
\text { choose the diagonal } \\
\text { loading factor, which } \\
\text { directly affects its } \\
\text { performance. }\end{array}$ \\
& & \\
\hline
\end{tabular}

\begin{tabular}{|c|c|c|}
\hline $\begin{array}{l}\text { Eigen-Space } \\
\text { Based }\end{array}$ & $\begin{array}{l}\text { Excellent } \\
\text { robustness } \\
\text { against } \\
\text { arbitrary } \\
\text { steering vector } \\
\text { errors. }\end{array}$ & $\begin{array}{l}\text { Degrade severely if } \\
\text { the low-rank } \\
\text { interference-plus- } \\
\text { signal assumption is } \\
\text { violated or if the } \\
\text { subspace dimension } L \\
\text { is uncertain or known } \\
\text { imprecisely }\end{array}$ \\
\hline LCMV & $\begin{array}{l}\text { Improved } \\
\text { robustness }\end{array}$ & $\begin{array}{l}\text { Strong degradation of } \\
\text { the output SINR }\end{array}$ \\
\hline $\begin{array}{l}\text { MVDR- } \\
\text { Minimum } \\
\text { Variance } \\
\text { Distortionless } \\
\text { Performance }\end{array}$ & $\begin{array}{l}\text { Gives } \\
\text { distortionless } \\
\text { performance in } \\
\text { the Direction } \\
\text { of Interest }\end{array}$ & $\begin{array}{l}\text { Unable to distinguish } \\
\text { between two closely } \\
\text { spaced plane waves }\end{array}$ \\
\hline Root MVDR & $\begin{array}{l}\text { Better } \\
\text { Performance }\end{array}$ & $\begin{array}{l}\text { Lesser threshold } \\
\text { compared to MVDR }\end{array}$ \\
\hline $\begin{array}{l}\text { MUSIC- } \\
\text { Multiple Signal } \\
\text { Classification } \\
\text { Algorithm }\end{array}$ & $\begin{array}{l}\text { High level of } \\
\text { orthogonality } \\
\text { between } \\
\text { signals. } \\
\text { Higher } \\
\text { resolution \& } \\
\text { accuracy }\end{array}$ & $\begin{array}{l}\text { Gives the } \\
\text { Pseudospectrum only. }\end{array}$ \\
\hline Root MUSIC & $\begin{array}{l}\text { Less } \\
\text { computational } \\
\text { time, higher } \\
\text { resolution }\end{array}$ & $\begin{array}{l}\text { Limited to linear } \\
\text { antennas, equispaced. }\end{array}$ \\
\hline $\begin{array}{l}\text { Unitary } \\
\text { MUSIC }\end{array}$ & $\begin{array}{l}\text { Less } \\
\text { Computational } \\
\text { complexity }\end{array}$ & $\begin{array}{l}\text { No much } \\
\text { performance } \\
\text { improvement from } \\
\text { Root MUSIC. }\end{array}$ \\
\hline $\begin{array}{l}\text { ESPIRIT- } \\
\text { Estimation of } \\
\text { signal } \\
\text { parameters via } \\
\text { rotational } \\
\text { invariance } \\
\text { technique }\end{array}$ & $\begin{array}{l}\text { No need of } \\
\text { searching the } \\
\text { maxima in } \\
\text { Pseudo } \\
\text { spectrum. } \\
\text { Less sensitive } \\
\text { to noise }\end{array}$ & More prone to errors. \\
\hline $\begin{array}{l}\text { Compressive } \\
\text { Sensing Based } \\
\text { Beamforming }\end{array}$ & $\begin{array}{l}\text { Less number of } \\
\text { measurements } \\
\text { Increased } \\
\text { resolution. }\end{array}$ & \\
\hline
\end{tabular}




\section{COMPRESSIVE SENSING FOR DOA ESTIMATION}

CS [39-41] is a recently developed mathematical framework, which asserts that a sparsely representable signal can be reconstructed using a small number of linear measurements. For example, consider a signal $\mathrm{x}$,

$$
x=\psi s
$$

which is k-sparse in the basis defined by the columns of $\psi$. According to CS, if non-traditional linear measurements in the form of randomized projections are taken,

$$
y=\varphi x
$$

The signal $\mathrm{x}$ can be exactly reconstructed with a high probability with a lesser number of samples, from the compressive measurements by solving a convex optimization problem subject to :

$$
y=\varphi \psi s
$$

which can be solved efficiently with linear programming The key result is that the required number of measurements is linked linearly to the sparsity-k of the signal.

The Compression is done at Sensing level, rather after the sensing. This leads to a greater reduction of samples, taking only fewer measurements, $\mathrm{M}$, with

$$
M=K \log (N)
$$

where, $\mathrm{K}$ is the sparsity in angle domain, $\mathrm{N}$ is the original number of samples used. The technique of Compressive Sensing can be used for DOA estimation, which consider only sparse number of samples.

In [42], a compressive wireless array is proposed for bearing estimation. In [43], a compressive beamforming method is presented. Both approaches apply compressive sampling in the time domain to reduce the ADC sampling rate or the number of time samples for each element of the array. In [44] the DOA estimation of narrowband sources impinging on a uniform circular array was considered. In [45] the formulation leads to second-order cone (SOC) programming where the optimization is performed over the entire signal space. The very high computational complexity of this formulation can be reduced by introducing the singular value decomposition (SVD) of the measured data matrix. The method in [46] tries to reconstruct the signals for sparse sources in the time domain with a combined $11-12$ norm minimization similar to [45]. In [47] a new hardware architecture exploiting compressive sensing (CS) for direction estimation is also used.

In [48], a Spatial Compressive Sensing (SCS) approach is proposed, the sensing and the reconstruction processes can be performed incrementally while improving the spatial spectrum estimation performance proportional to an increase in array orientation diversity (the number of array orientations). Finally, array orientation diversity is proposed to address some of the challenging problems that arise in passive sonar applications, such as: low bearing resolution when using short arrays, incoherency between sensors when using long arrays, poor estimation performance at endfire, short samples support when the temporal coherency is limited by the motion of the array or sources, spatial correlation of the ambient noise and the correlation among sources etc. The work shows that array orientation diversity provides improvement in spatial spectrum estimation which is not associated with a linear increase in the number of spatial measurements.

The compressive bearing estimation approach based on spatial sparsity has several advantages over other approaches in the literature, such as MVDR, MUSIC, and previous methods using sparsity, which require Nyquist sampling at the sensors [48].
Creating a bearing spectrum with many fewer measurements decreases the communication load in wireless networks and enables lower data acquisition rates, which might be very important for high bandwidth applications. Moreover, the array geometry can be arbitrary but known. Other advantages include increased resolution and robust to noise. A considerable reduction in hardware costs may also be achieved.

\section{CONCLUSION}

Estimating the direction-of-arrival (DOA) of propagating plane waves is a problem of broad interest in a variety of fields including wireless communications, radar and sonar systems, acoustic signal processing, medical imaging and seismology. Through this review, a detailed survey on various DOA estimation beamforming algorithms existing, were made. Based on the literature survey made, it may be concluded that beamforming based on Compressive Sensing for DOA estimation is more advantageous. As the signal of interest, here is a sparse signal, it is advisable to switch on to Compressive sensing based beamforming for DOA estimation which requires only a fewer number of samples, rather than the Nyquist sampling. Compressive sensing based beamforming called Compressive Beamforming for DOA estimation was found to be more beneficial over Nyquist sampling.

\section{REFERENCES}

[1] Barry D. Van Veen and Kevin M. Buckley, "Beamforming: A Versatile Approach to Spatial Filtering”, IEEE ASSP magazine April 1988.

[2] H. Van Trees, Optimum Array Processing. New York: Wiley, 2002, sec. Part IV of "Detection, Estimation and Modulation Theory".

[3] Mestre and Lagunas': "Finite Sample Size Effect on Minimum Variance Beamformers: Optimum Diagonal Loading Factor for Large Arrays" IEEE Trans. Signal Processing, Vol. 54, No. 1, Jan 2006.

[4] H. Cox, R. M. Zeskind, and M. H. Owen, "Robust adaptive beamforming," IEEE Trans. Acoust., Speech, Signal Processing, vol. 35, Oct. 1987.

[5] B. D. Carlson, "Covariance matrix estimation errors and diagonal loading in adaptive arrays," IEEE Trans. Aerosp. Electron. Syst., vol. 24, July 1988.

[6] L. Chang and C. C. Yeh, "Performance of DMI and eigenspace-based beamformers," IEEE Trans. Antennas Propagation, vol. 40, Nov. 1992.

[7] M.H. Er and A. Cantoni, "Derivative constraints for broadband element space antenna array processors," IEEE Transactions on Antennas and Propagation, vol. ASSP-31, no. 6, December 1983.

[8] O. L. Frost III, "An algorithm for linearly constrained adaptive processing,” Proc. IEEE, vol. 60, Aug. 1972.

[9] A.B. Gershman, G.V. Serebryakov, and J. F.Bohme, "Constrained Hung-Turner adaptive beamforming algorithm with additional robustness to wideband and moving jammers,"IEEE Transactions on Antennas and Propagation, vol. AP-44,March 1996.

[10] R. Li, X. Zhao, and X. W. Shi "Derivative Constrained Robust LCMV Beamforming 
Algorithm", Progress In Electromagnetics Research C, Vol. 4, 2008.

[11] S. Shahbazpanahi, A. B. Gershman, Z.-Q. Luo, and K. M. Wong, "Robust adaptive beamforming for general-rank signal models," IEEE Trans. Signal Processing,Vol. 51, Sept. 2003.

[12] R. G. Lorenz and S. P. Boyd, "Robust minimum variance beamforming," IEEE Trans. Signal Processing, vol. 53, May 2005.

[13] J. Li, P. Stoica, and Z. Wang. On robust capon beamforming and diagonal loading. IEEE Trans. SignalProc., 51, July 2003.

[14] J. Li, P. Stoica, and Z. Wang. Doubly constrained robust capon beamformer. IEEE Trans. Signal Processing, 52(9): Sep. 2004.

[15] Xin Song, Jinkuan Wang, Bin Wang, Yinghua Han, "Robust Adaptive Beamforming under in the Presence of Mismatches", Proceedings of the IEEE International Conference on Automation and Logistics, August 2009..

[16] E. W. Vook and R. T. Compton, Jr., "Bandwidth performance of linear adaptive arrays with tapped delay-line processing," IEEE Transactions on Aerospace and Electronic Systems, vol. 28, no. 3, July 1992.

[17] M. Rubsamen and A.B. Gershman, "Robust presteered broadband beamforming based on worstcase performance optimization," in Proc. IEEE Workshop on Sensor Array and Multichannel Signal Processing, July 2008.

[18] Yong Zhao, Wei Liu; Langley, R.J, "Robust Broadband Beamforming Based on Frequency Invariance Constraints and worst-case Performance Optimization", Proceedings of the 4th International Symposium on Communications, Control and Signal Processing, March 2010.

[19] Yong Zhao, Wei Liu; Langley, R.J, “Adaptive Wideband Beamforming With Frequency Invariance Constraints", IEEE Transactions on Antennas and Propagation, Volume:59, Issue 4,April 2011

[20] J. Capon, “.High resolution n frequency wavenumber spectrum nalysis" Proc. IEEE, August 1969.

[21] R. T. Lacoss. Data adaptive spectral analysis methods. Geophysics, 01.36, p. 661, 1971.

[22] Vaidhyanathan \& Buckley, " Performance analysis of MVDR spatial spectrum estimator", IEEE transactions on Signal processing, June 1995.

[23] V U. Reddy, A. Paulraj, and T. Kailath, "Performance analysis of the optimum beamformer in the presence of correlated sources and its behavior under spatial smoothing," IEEE Trans. Acoust., Speech, Signal Processing, vol. ASSP-35, July 1987.

[24] K J. Raghunath, and V. U Reddy, "Finite Data Performance Analysis of MVDR Beamformer with and without Spatial Smoothing" IEEE Transactions On Signal Processing, Vol. 40, NO. 2 , Nov 1992.

[25] Vorobyov, S., Gershman, A., and Luo, Z.: 'Robust adaptive beamforming using worst-case performance optimization: A solution to the signal mismatch problem', IEEE Trans. Signal Process., vol 51,No.2, , Feb 2003.

[26] S. A. Vorobyov, A. B. Gershman, Z-Q. Luo, and N. $\mathrm{Ma}$, "Adaptive beamforming with joint robustness against mismatched signal steering vector and interference nonstationarity,'IEEE Signal Processing Lett., vol. 11, Feb. 2004.

[27] R. 0. Schmidt, “ Multiple emitter location and signal parameter estimation" IEEE Trans. Antennas Propag., vol.AP-34, pp. 276-280, March 1986.

[28] G. Bienvenu and L. Kopp., "Optimality of high resolution array processing using the eigensystem approach", IEEE Trans. Acoust., Speech, Signal rocess, vol.ASSP-31, pp. 1234-1248, October 1983

[29] B. D. Rao and K. V. S. Hari, " Performance analysis of root-MUSIC" IEEE Trans. Acous t., Speech, Signal Process., vol.ASSP-37, pp. 1939-1949, December 1989.

[30] M. Pesavento, A. B. Gershman, and M. Haardt, ".Unitary MUSIC with real valued eigen decomposition: A theoretical and experimental study", IEEE Trans, signal process, May 2000

[31] R. Roy and T. Kailath, " ESPRIT: Estimation of signal parameters via rotational invariance techniques" , IEEE Trans. Acoust., Speech, Signal Process, vol.ASSP-37, pp. 984-995, July 1989.

[32] R. Roy, " ESPRIT: Estimation of Signal Parameters via Rotational Invariance Techniques", Ph.D. Dissertation, Stanford University, Stanford, California, 1987.

[33] X. L. Xu and K. M. Buckley, "Bias analysis of the MUSIC location estimator", IEEE Trans. Acoust., Speech, Signal Process., vol.ASSP-40, pp. 2559-2569, October 1992.

[34] Lotfi Osman, " Comparative Study of HighResolution Direction-of-Arrival Estimation Algorithms for Array Antenna System", International Journal of Research and Reviews in Wireless Communications (IJRRWC) Vol. 2, No. 1, March 2012.

[35] R. Roy, A. Paulraj, and T. Kailath,. " ESPRIT-A subspace rotation approach to estimation of parameters of cisoids in noise", IEEE Trans. Acoust., Speech, Signal Process., vol.ASSP-34, pp. 1340-1342, October 1986.

[36] R. Roy and T. Kailath, "ESPRIT-Estimation of signal parameters via rotational invariance techniques", In E. F. Deprettere, editor, Singular Value Decomposition and Signal Processing North-Holland, Amsterdam, The Netherlands, September 1987.

[37] Jihao Yin and Tianqi Chen, "Direction of Arrival Estimation Using a Sparse Representation of Array Covariance Vectors", IEEE Transactions on Signal processing, Vol. 59, No. 9, September 2011.

[38] D Malioutov, M Cetin and AS Willsky, "A Sparse signal reconstruction perspective for source localization with sensor arrays", IEEE Transactions on Signal processing, vol. 53, no 8, Aug 2005.

[39] D. Donoho, "Compressed sensing”, IEEE Trans. on Information Theory, pp. 1289-1306, Apr. 2006. 
[40] R. Baraniuk and P. Steeghs, "Compressive radar imaging," in IEEE Radar Conf., 2007, pp. 128-133.

[41] Emmanuel J. Candès and Michael B. Wakin, "An introduction to Compressive Sampling “, IEEE signal processing Magazine, March 2008.

[42] V. Cevher, A. Gurbuz, J. McClellan, and R. Chellappa, "Compressive wireless arrays for bearing estimation", ICASSP 2008, pp. 2497-2500, March 31 2008-April 4, 2008.

[43] A. Gurbuz, J. McClellan, and V. Cevher, "A compressive beamforming method", ICASSP 2008, pp. 2617-2620, March 31 2008-April 4, 2008.

[44] Fuchs, J. J, "On the application of the global matched filter to DOA estimation with uniform circular arrays." IEEE Transactions on Signal Processing, 49 (2001).
[45] Malioutov, D., Cetin, M., and Willsky, “ A sparse signal reconstruction perspective for source localization with sensor arrays." IEEE Transactions on Signal Processing, 53, 8 (2005), 3010-3022.

[46] Model, D. and Zibulevsky M. "Signal reconstruction in sensor arrays using sparse representations." Signal Processing, 86 (2006), 624-638.

[47] Wang, Y., Leus, G., and Pandharipande, "A. Direction estimation using compressive sampling array processing.", In Proceedings of the IEEE/SP 15th Workshop on Statistical Signal Processing, Aug. 2009, 626-629.

[48] I.Bilik, "Spatial Compressive Sensing for Directionof-Arrival Estimation of Multiple Sources using Dynamic Sensor Arrays” IEEE Proceedings 2011. 